\title{
Amino Acid Functionalized Dendritic Polyglycerol for Safe and Effective siRNA Delivery
}

Hanxiang Zeng, ${ }^{+}$Cathleen Schlesener, ${ }^{\ddagger}$ Olivia Cromwell, ${ }^{+}$Markus Hellmund, ${ }^{\ddagger}$ Rainer Haag ${ }^{*, \neq}$, Zhibin Guan $^{*,+}$

${ }^{\dagger}$ Department of Chemistry, 1102 Natural Sciences 2, University of California, Irvine, California 92697-2025, United States

${ }^{\ddagger}$ Institut für Chemie und Biochemie, Freie Universität Berlin, Takustrasse 3, 14195, Berlin, Germany

Supporting Information

1. List of Amino Acid Functionalized dPG

2. ${ }^{1}$ H NMR Assignment and Calculation of Functionalization Ratio

3. Additional Electrophoresis Data

4. Initial Transfection Screening Results 
1. List of Amino Acid Functionalized dPG in this study.

Table S1. dPG-AA samples and their functionalization ratio determined by ${ }^{1} \mathrm{H}$ NMR.

\begin{tabular}{|c|c|c|c|}
\hline \multirow{2}{*}{ Sample Name } & \multirow{2}{*}{ Amine Density } & \multicolumn{2}{|c|}{ Functionalization Ratio } \\
\cline { 3 - 4 } & & histidine & aromatics amino acid \\
\hline $14 \mathrm{kD}-70 \%-75 \mathrm{H} 25 \mathrm{~F}$ & $68 \%$ & $68 \%$ & $25 \%$ \\
\hline $14 \mathrm{kD}-70 \%-75 \mathrm{H} 25 \mathrm{Y}$ & $68 \%$ & $65 \%$ & $25 \%$ \\
\hline $14 \mathrm{kD}-70 \%-75 \mathrm{H} 25 \mathrm{~W}$ & $68 \%$ & $59 \%$ & $24 \%$ \\
\hline $14 \mathrm{kD}-70 \%-90 \mathrm{H} 10 \mathrm{~W}$ & $68 \%$ & $81 \%$ & $10 \%$ \\
\hline $14 \mathrm{kD}-70 \%-60 \mathrm{H} 40 \mathrm{~W}$ & $68 \%$ & $55 \%$ & $40 \%$ \\
\hline $14 \mathrm{kD}-50 \%-75 \mathrm{H} 25 \mathrm{~W}$ & $49 \%$ & $64 \%$ & $30 \%$ \\
\hline $14 \mathrm{kD}-50 \%-60 \mathrm{H} 40 \mathrm{~W}$ & $49 \%$ & $58 \%$ & $38 \%$ \\
\hline $14 \mathrm{kD}-35 \%-75 \mathrm{H} 25 \mathrm{~W}$ & $39 \%$ & $71 \%$ & $24 \%$ \\
\hline $14 \mathrm{kD}-35 \%-60 \mathrm{H} 40 \mathrm{~W}$ & $39 \%$ & $58 \%$ & $42 \%$ \\
\hline $55 \mathrm{kD}-35 \%-75 \mathrm{H} 25 \mathrm{~W}$ & $38 \%$ & $70 \%$ & $27 \%$ \\
\hline $55 \mathrm{kD}-35 \%-60 \mathrm{H} 40 \mathrm{~W}$ & $38 \%$ & $60 \%$ & $40 \%$ \\
\hline
\end{tabular}




\section{2. ${ }^{1} \mathrm{H}$ NMR Assignment and Calculation of Functionalization Ratio}

The NMR spectrum of $14 \mathrm{kD}-70 \%-60 \mathrm{H} 40 \mathrm{~W}$ was used as an example for the assignment. The NMR spectra of all the other dPG-AAs were very similar, differing only in the integration values of the various peaks. The calculation of the actual functionalization ratio was carried out as follows:

Peak $\sim 8.5$ contains $1 \mathrm{H}$ from His; integration set to 1.00

Peak from 7.0 7.5 (combined peak area, 4.60) contain $1 \mathrm{H}$ from histidine and $5 \mathrm{Hs}$ from tryptophan, therefore

Molar amount of $\operatorname{Trp}=(4.60-1.00) / 5=0.72$.

Both His and Trp contribute $3 \mathrm{Hs}$ in the region 2.5 4.5 (combined peak area, 2.27+16.30), therefore

Amount of Hs from dPG core $=2.27+16.30-1 * 3-0.72 * 3=13.41$

And each repeating unit of dPG has 5 Hs in the region 2.5 4.5, therefore

Molar amount of dPG repeating units $=13.41 / 5=2.68$

Only $68 \%$ of dPG repeating units were converted to amines, therefore

Molar amount of amines $=2.68 * 0.68=1.82$

Functionalization ratio:

His $\%=1 / 1.82=55 \%$

$\operatorname{Trp} \%=0.72 / 1.82=40 \%$ 


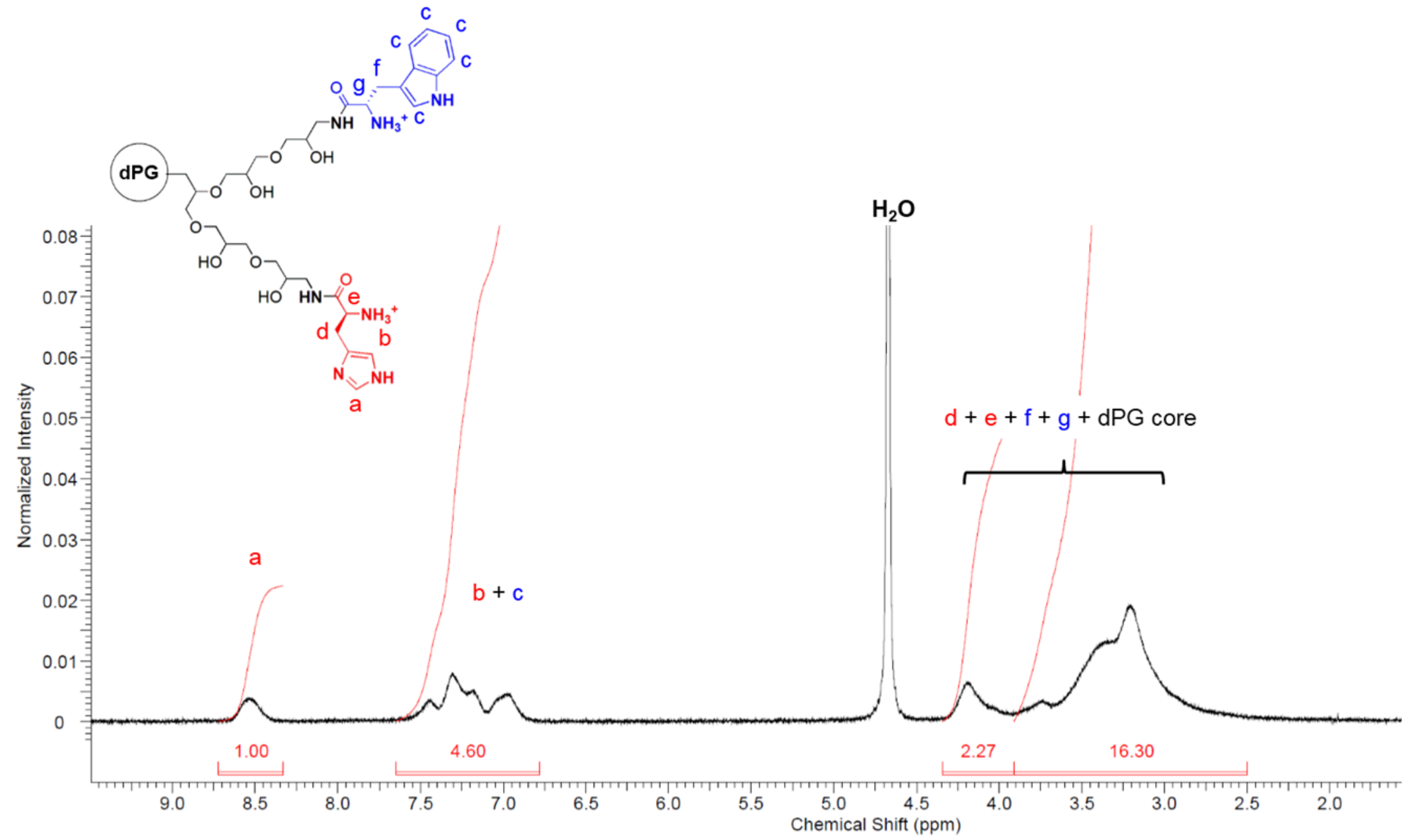

Figure S1. Representative ${ }^{1} \mathrm{H}$ NMR assignment of functionalized dPG-AA (14kD-70\%-60H40W) 


\section{Additional Gel Electrophoresis Images of dPG-AA/siRNA Complexation}
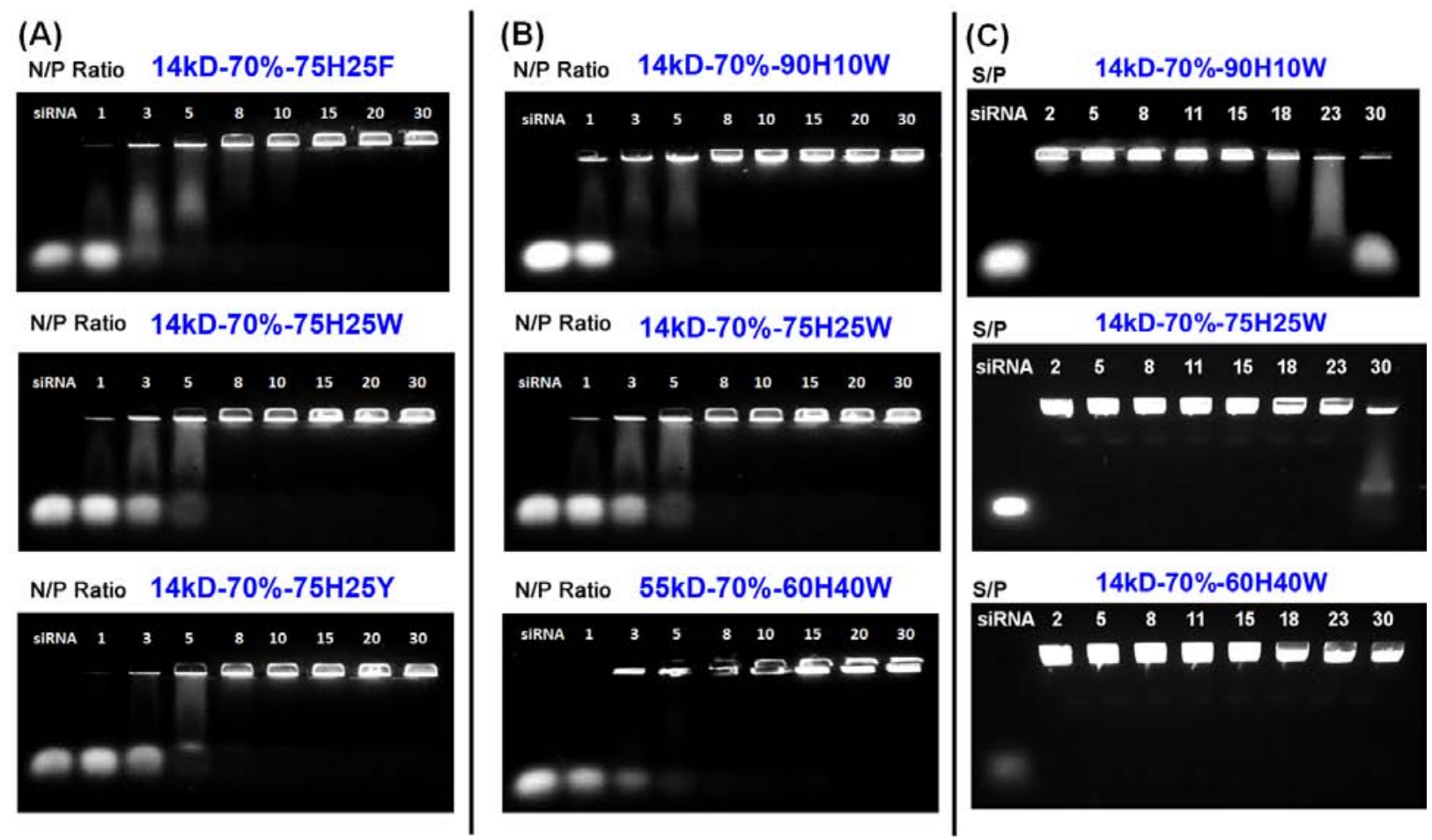

Figure S2. Additional gel electrophoresis images. (A) - (B) Complexation of different dPG-AA samples with siRNA. (C) Dextran Sulfate competitive binding assay of dPG-AAs with different Trp incorporation. 


\section{Initial Transfection Screening Results.}

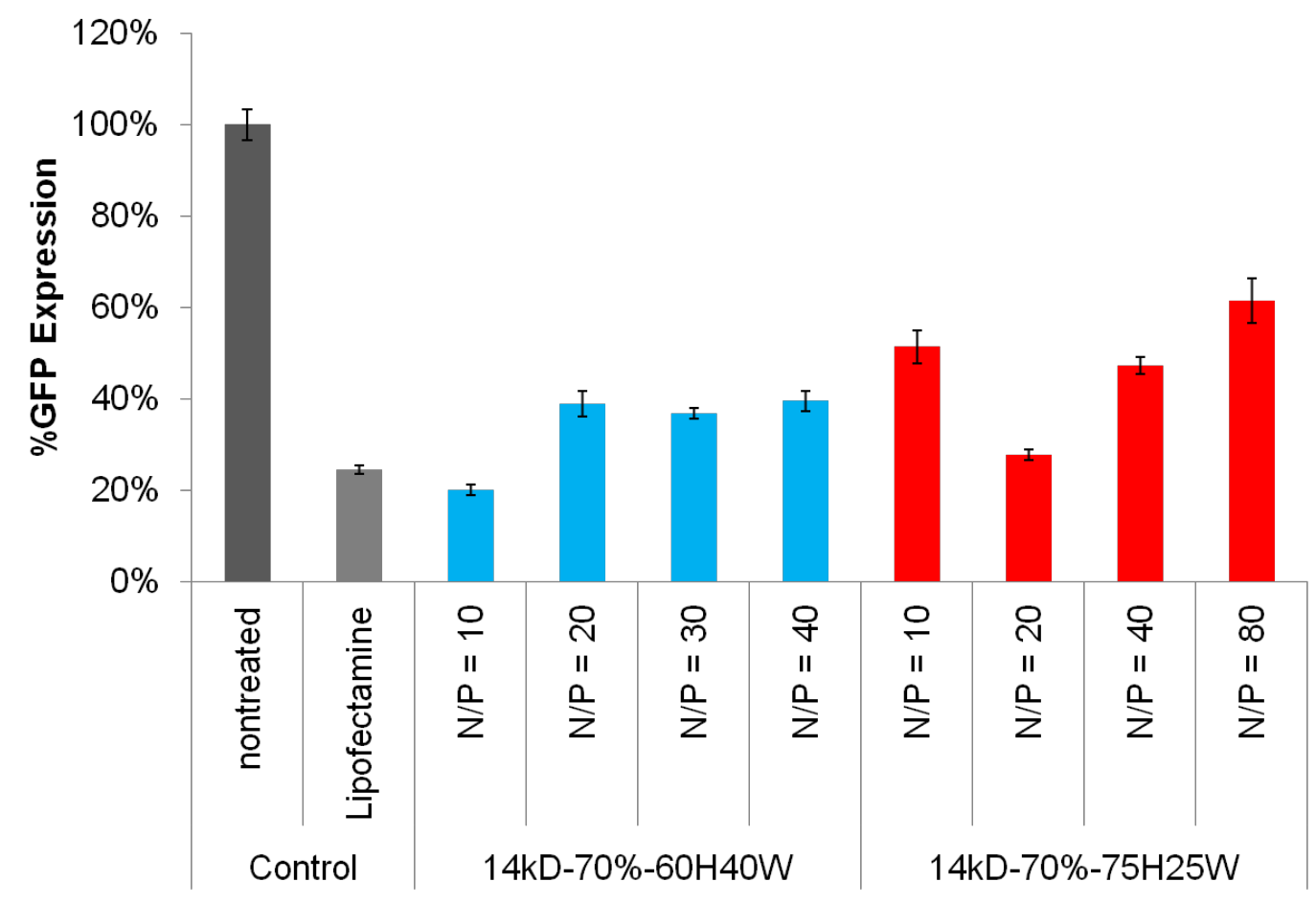

Figure S3. Transfection results of dPG-AAs at different N/P ratios. Transfection was tested in NIH 3 T3 cell line with 100 nM siRNA concentration. The GFP fluorescence was quantified by flow cytometry. 